University of Windsor

Scholarship at UWindsor

Spring 2011

\title{
An OWA-TOPSIS method for multiple criteria decision analysis
}

Ye Chen

Kevin W. Li Dr.

University of Windsor

Si-feng Liu

Follow this and additional works at: https://scholar.uwindsor.ca/odettepub

Part of the Business Commons

\section{Recommended Citation}

Chen, Ye; Li, Kevin W. Dr.; and Liu, Si-feng. (2011). An OWA-TOPSIS method for multiple criteria decision analysis. Expert Systems with Applications, 38 (5), 5205-5211.

https://scholar.uwindsor.ca/odettepub/60

This Article is brought to you for free and open access by the Odette School of Business at Scholarship at UWindsor. It has been accepted for inclusion in Odette School of Business Publications by an authorized administrator of Scholarship at UWindsor. For more information, please contact scholarship@uwindsor.ca. 


\title{
An OWA-TOPSIS method for multiple criteria decision analysis
}

\author{
Ye Chen ${ }^{a *}$, Kevin W. Li $^{b}$, Si-feng Liu ${ }^{a}$ \\ ${ }^{a}$ College of Economics and Management, Nanjing University of Aeronautics and \\ Astronautics, Najing, Jiangsu, 210016, China \\ ${ }^{b}$ Odette School of Business, University of Windsor, Windsor, Ontario, N9B 3P4, Canada
}

\begin{abstract}
A hybrid approach integrating OWA (Ordered Weighted Averaging) aggregation into TOPSIS (technique for order performance by similarity to ideal solution) is proposed to tackle multiple criteria decision analysis (MCDA) problems. First, the setting of extreme points (ideal and anti-ideal points) in TOPSIS is redefined and extended for handling the multiple extreme points situation where a decision maker (DM) or multiple DMs can provide more than one pair of extreme points. Next, three different aggregation schemes are designed to integrate OWA into the TOPSIS analysis procedure. A numerical example is provided to demonstrate the proposed approach and the results are compared for different aggregation settings and confirm the robustness of rankings from different scenarios.
\end{abstract}

Key words: Multiple criteria decision analysis, TOPSIS, OWA, distance-based ranking, decision aggregation

\section{Introduction}

Due to ever increasing complexity of human society, people often need to consider multiple criteria (attributes, factors, objectives) to make decisions. The research area of multiple criteria decision analysis (MCDA) is developed to provide decision aid for complex decision situations. MCDA aims to furnish a set of decision analysis techniques to help decision makers (DMs) logically identify, compare, and evaluate alternatives according to diverse, usually conflicting, criteria arising from societal, economic, and environmental considerations. This body of literature has also been interexchangeably referred to as multiple criteria (attribute) decision aid (making) [11].

\footnotetext{
* Corresponding author. E-mail:chenye@nuaa.edu.cn;Phone:+86-25-84895760;Fax:+86-2584895760 .
} 
MCDA provides a systematic framework to investigate complex decision problems containing multiple intertwining criteria. MCDA concentrates on decision analysis with a finite set of alternatives and offers a host of methods for preference elicitation and aggregation. A unique feature of MCDA is preference-based aggregation. To reach a final recommendation, it is inevitable that an aggregation procedure is required to synthesize alternatives' performances over different criteria. To achieve this more effectively, the aggregation in MCDA is based on DMs' preferences instead of relying on traditional cost-benefit analysis in which all criteria have to be converted to monetised measures [10].

Roy [21] suggests three problématiques (fundamental problems) for MCDA, whereby a set of alternatives, $\mathbf{A}$, is evaluated to produce a final decision result:

- Choice. Choose the best alternative from A.

- Sorting. Sort the alternatives of A into relatively homogeneous groups in a preference order.

- Ranking. Rank the alternatives of $\mathbf{A}$ from best to worst.

Among the above three types of decision problems, ranking produces the most comprehensive information with a full preference order of alternatives. Obviously, the best alternative (choice) can be conveniently identified if a full ranking is obtained. Also, a sorting problem can be addressed by applying a logical assignment procedure to the generated ranking results [4]. Various MCDA approaches are developed to handle different types of MCDA problems, including multiattribute utility theory (MAUT) [18], outranking methods [21] and analytic hierarchy process (AHP) [22], to name a few. A recent state-of-the-art review of MCDA [11] summarizes a wide variety of MCDA approaches.

The TOPSIS (technique for order performance by similarity to ideal solution) method [13] constitutes a useful technique in solving ranking problems. The basic idea of the TOPSIS is simple and intuitive: measure alternatives' distances to pre-defined ideal and anti-ideal points first and, then, aggregate the separate distance information to reach overall evaluation results. Some features of TOPSIS, as summarized in [19,23], include clear and easily understandable geometric meaning, simultaneously consideration from both best and worst points of view, and convenient calculation and implementation. Different methods have thus been developed to extend the original TOPSIS idea $[2,3$, $9,14,16,20,23,25]$.

The Ordered Weighted Averaging aggregation operators, commonly known as OWA operators, are introduced by Yager [28] to provide a parameterized class of mean-type aggregation operators. Many notable mean operators, such as the Max, arithmetic average, median, and Min, are members of this class. OWA operators have been widely used in computational intelligence due to their flexibility in modeling linguistically expressed aggregation instructions [8]. A comprehensive literature review and summary of OWA operators with diverse applications is provided in $[24,30]$. 
TOPSIS and OWA methods become increasingly popular research topic in several academic fields. For example, within the journal of Expert Systems with Applications, a search on the keywords "TOPSIS" through ScienceDirect identifies 105 papers. Especially there is a significant increase in 2009: over 80 papers has been published or accepted for publication. In this paper a hybrid approach of OWA aggregation and TOPSIS is designed to incorporate the unique features from both methods to provide additional flexibility for MCDA. The remainder of the paper is organized as follows: overviews of MCDA, OWA, and TOPSIS are given in Section 2; next, in Section 3 a hybrid method, integrating the OWA aggregation into the TOPSIS, is constructed and explained in detail; then, Section 4 presents a numerical example adapted from [23] to demonstrate the proposed method and, finally, some concluding remarks are furnished in Section 5.

\section{Overviews of MCDA, OWA and TOPSIS}

\subsection{An overview of $M C D A$}

The analysis of an MCDA problem can be summarized as the following three steps [7]: (1) Problem construction, in which the DM's objectives are defined, all possible alternatives are identified, and criteria are determined whereby successes in achieving the objectives are measured; (2) Preference elicitation and aggregation, in which the DM's preferences within and across criteria are obtained and aggregated; (3) Implementation, in which a constructed preference model is utilized to evaluate all alternatives, thereby the 'problématique' selected by the DM can be solved. The analysis results can be employed as an aid to the actual decision making process.

Step (1) aims to structure an MCDA problem. Let the set of alternatives be $\mathbf{A}=$ $\left\{a^{1}, \cdots, a^{i}, \cdots, a^{|\mathbf{A}|}\right\}$ and the set of criteria be $\mathbf{C}=\left\{c_{1}, \cdots, c_{j}, \cdots, c_{\mid \mathbf{C}}\right\}$, where $|\mathbf{X}|$ represents the cardinality of a set $\mathbf{X}$. When step (1) is completed, the consequence of alternative $a^{i}$ on criterion $c_{j}$, denoted by $m_{j}^{i}$, will be measured for every $i=1, \ldots,|\mathbf{A}|$ and $j=1, \ldots,|\mathbf{C}|$, constituting the $(i, j)$-entry of a $|\mathbf{A}| \times|\mathbf{C}|$ matrix called the information (or performance) matrix. The structure of this matrix is shown in Figure 1. Note that a consequence is a direct measurement of an alternative according to a criterion (e.g. cost in dollars). Generally speaking, a consequence is an objective physical measurement.

The DM's preferences are crucial in reaching a final recommendation for an MCDA problem, and different approaches to modeling preferences of the same problem may lead to different conclusions. Formally, as we interpret MCDA procedures, a DM may have preferences on consequences, called values, and preferences over criteria, referred to as weights.

Preferences on consequences, or "values," are refined data obtained by processing conse- 


\begin{tabular}{|c|c|c|c|c|c|}
\hline & \multicolumn{4}{|c|}{ Alternatives } \\
\hline & & $a^{1}$ & $a^{2}$ & $\cdots$ & $a^{|\mathbf{A}|}$ \\
\hline \multirow{4}{*}{ 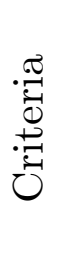 } & $c_{1}$ & & & & \\
\hline & $c_{2}$ & & & $\downarrow$ & \\
\hline & $:$ & -- & $\rightarrow$ & $m_{j}^{i}$ & \\
\hline & $C_{|\mathbf{C}|}$ & & & & \\
\hline
\end{tabular}

Fig. 1. Performance Matrix in MCDA, adapted from [7]

quences (original and raw information) according to the needs and objectives of the DM. This is a necessary step to convert and normalize consequences into a common comparative ground as consequences on different criteria often assume significantly different formats. The general relationship between consequences and values can be expressed as a mapping from consequences to values, $v_{j}^{i}=f_{j}\left(m_{j}^{i}\right)$, where $v_{j}\left(a^{i}\right)$ and $m_{j}^{i}$ are a value and a consequence measurement, respectively. The DM's values over all criteria for alternative $a^{i}$ constitute a value vector, $\mathbf{v}\left(a^{i}\right)=\left(v_{1}\left(a^{i}\right), \cdots, v_{|\mathbf{C}|}\left(a^{i}\right)\right)$. It is often assumed that criteria are preference monotonic along consequences: (1) benefit criteria: the larger the consequence value, the better; (2) cost criteria: the smaller the consequence value, the better.

Preferences on criteria, or "weights," refer to expressions of the relative importance of criteria. The weight for criterion $c_{j} \in \mathbf{C}$ is denoted by $w_{j} \in \mathbb{R}^{+}$. Usually it is required that $\sum_{j=1}^{|\mathbf{C}|} w_{j}=1$, and the DM's weight vector is denoted by $\mathbf{w}=\left(w_{1}, \cdots, w_{j}, \cdots, w_{|\mathbf{C}|}\right)$.

After an MCDA problem is structured and preferences are obtained, a global model is required to aggregate preferences and solve the specified problématique. For $a^{i} \in \mathbf{A}$, the overall evaluation of alternative $a^{i}$ is denoted by $V\left(a^{i}\right) \in \mathbb{R}$, where $V\left(a^{i}\right)=F\left(\mathbf{v}\left(a^{i}\right), \mathbf{w}\right)$. Here, $F(\cdot)$ is a real-valued mapping from the value vector $\mathbf{v}\left(a^{i}\right)$ and the weight vector $\mathbf{w}$ to a numerical evaluation of $a^{i}$. A typical example is the linear additive value function, $V\left(a^{i}\right)=\sum_{j=1}^{|\mathbf{C}|} w_{j} \cdot v_{j}\left(a^{i}\right)[13]$.

\subsection{OWA aggregation operators}

An OWA operator is a process to aggregate a set of data, $\mathbf{B}=\left\{b^{1}, \cdots, b^{|\mathbf{B}|}\right\}$, into a representative datum, i.e. $\mathbb{R}^{|\mathbf{B}|} \rightarrow \mathbb{R}$, with an associated weight vector $\mathbf{Q}=\left(q_{1}, \cdots, q_{|\mathbf{B}|}\right)$, $(|\mathbf{B}|=|\mathbf{Q}|)$ such that $\sum_{j=1}^{|\mathbf{B}|} q_{j}=1,0 \leq q_{j} \leq 1$, and $O W A_{|\mathbf{Q}|}\left(b^{1}, \cdots, b^{|\mathbf{B}|}\right)=\sum_{i=1}^{|\mathbf{B}|} q_{j} b^{\sigma(j)}$, where $\{\sigma(1), \cdots, \sigma(|\mathbf{B}|)\}$ is a permutation of $\{1, \cdots,|\mathbf{B}|\}$ such that $b^{\sigma(j-1)} \geq b^{\sigma(j)}$, for all $j=\{2, \cdots,|\mathbf{B}|\}$, i.e. $b^{\sigma(j)}$ is the $j$ th largest element in $\mathbf{B}$ [24]. Hence, an important 
feature of OWA operators is the re-ordering of the elements that makes it a nonlinear operator, and the vector of $\mathbf{Q}$ is not the representation of relative importance of different types of information in $\mathbf{B}$, but a mechanism to smoothly achieve any kind of averaging between Max and Min for $\mathbf{B}$.

Two important features called the dispersion (entropy) and the "orness" are defined as $\operatorname{Disp}(\mathbf{Q})=-\sum_{i=1}^{|\mathbf{B}|} q_{j} \ln q_{j}$ and $\operatorname{orness}(\mathbf{Q})=\frac{1}{|\mathbf{B}|-1} \sum_{j=1}^{|\mathbf{B}|}(|\mathbf{B}|-j) q_{j}$, respectively [28]. The dispersion gauges the degree to which all data are equally aggregated. The orness is a value between 0 and 1 that represents the degree to which the aggregation is like an "OR" operation, and can be viewed as an optimism indicator of a decision maker. Some well-known averaging decision rules can be expressed as OWA operations below [26]:

- $O W A^{*}$ : Set $\mathbf{Q}=(1,0, \cdots, 0)$, then $O W A^{*}\left(b^{1}, \cdots, b^{|\mathbf{B}|}\right)=\max _{i=1}^{|\mathbf{B}|}\left(b^{i}\right)$, representing the most optimistic decision (maximax, "OR" decision) and $\operatorname{orness}(\mathbf{Q})=1$;

- $O W A_{*}$ : Set $\mathbf{Q}=(0,0, \cdots, 1)$, then $O W A_{*}\left(b^{1}, \cdots, b^{|\mathbf{B}|}\right)=\min _{i=1}^{|\mathbf{B}|}\left(b^{i}\right)$, representing the most pessimistic decision (minimin, "AND" decision) and orness $(\mathbf{Q})=0$;

- $O W A_{\text {average }}$ : Set $\mathbf{Q}=(1 /|\mathbf{B}|, \ldots, 1 /|\mathbf{B}|)$, then $O W A_{\text {average }}\left(b^{1}, \cdots, b^{|\mathbf{B}|}\right)=\frac{1}{|\mathbf{B}|} \sum_{i=1}^{|\mathbf{B}|} b^{i}$, yielding the equally likely decision and $\operatorname{orness}(\mathbf{Q})=0.5$.

Various approaches have been suggested for OWA weight generation, which can be generally divided into two categories: fuzzy linguistic quantifier approaches and optimization-based methods. Fuzzy linguistic quantifier approaches focus on the construction of regular increasing monotonic functions and the utilization of fuzzy linguistic quantifiers to obtain different weight information $[17,29,30]$. Optimization-based methods aim to incorporate optimization into determining the ordered weights by maximizing the entropy value of ordered weights or minimizing the variance of ordered weights under a given level of orness [15,26,27]. In this paper, a fuzzy linguistic quantifier approach is utilized due to its easy-to-understand meaning. The details are provided in Section 4.3.

\subsection{The TOPSIS method}

The TOPSIS analysis procedure can be summarized as the following steps [23]:

- Construct a performance matrix: establish a $|\mathbf{A}| \times|\mathbf{C}|$ matrix called the performance (information, decision) matrix as shown in Figure 1.

- Normalize the performance matrix: Apply a normalization process to the performance matrix to convert the original consequence data to values. Assume that $\forall m_{j}^{i} \in \mathbb{R}^{+}$, three widely used normalization functions, mapping from $m_{j}^{i}$ to $v_{j}^{i}\left(0 \leq v_{j}^{i} \leq 1\right)$, are listed below $[5,6,23]$ : 
(1) Vector normalization: $v_{j}^{i}=\frac{m_{j}^{i}}{\sqrt{\sum_{i=1}^{|\mathbf{A}|}\left(m_{j}^{i}\right)^{2}}}$;

(2) Sum-based normalization: $v_{j}^{i}=\frac{m_{j}^{i}}{\sum_{i=1}^{|\mathbf{A}|}\left(m_{j}^{i}\right)}$.

(3) Min-Max-based normalization: $v_{j}^{i}=\frac{m_{j}^{i}}{\max _{i=1}^{|\mathbf{A}|} m_{j}^{i}}\left(c_{j}\right.$ is a benefit criterion) and $v_{j}^{i}=$ $\frac{\min _{i=1}^{|\mathbf{A}|} m_{j}^{i}}{m_{j}^{i}}\left(c_{j}\right.$ is a cost criterion $)$

- Define ideal and anti-ideal points: Set an ideal point, $a^{+}$, and an anti-ideal point, $a^{-}$, as the combination of maximum or minimum values of $v_{j}\left(a^{i}\right)$ for all $a^{i} \in \mathbf{A}$ and $c_{j} \in$ $\mathbf{C}$, respectively. For example, for a benefit criterion $c_{j}$, using vector normalization, $v_{j}\left(a^{+}\right)=\max _{i=1}^{|\mathbf{A}|} v_{j}^{i}$ and $v_{j}\left(a^{-}\right)=\min _{i=1}^{|\mathbf{A}|} v_{j}^{i}$.

- Assign weights to criteria: Set $w_{j}\left(w_{j} \in \mathbb{R}^{+}\right.$and $\left.\sum_{j=1}^{|\mathbf{C}|} w_{j}=1\right)$ to represent the relative importance of the criterion, $c_{j}$.

- Calculate the distances from $a^{i}$ to $a^{+}$and $a^{-}$: the $p$-norm distance functions,

$$
D\left(a^{i}\right)^{+}=\left\{\sum_{j=1}^{|\mathbf{C}|} w_{j} \mid\left(v_{j}\left(a^{+}\right)-\left.v_{j}\left(a^{i}\right)\right|^{p}\right\}^{1 / p},\right.
$$

and

$$
D\left(a^{i}\right)^{-}=\left\{\sum_{j=1}^{|\mathbf{C}|} w_{j} \mid\left(v_{j}\left(a^{i}\right)-\left.v_{j}\left(a^{-}\right)\right|^{p}\right\}^{1 / p},\right.
$$

are often employed, where $p$ is a pre-defined distance norm, usually set as 1 or 2 ; and $|x|$ represents the absolute value of $x$.

- Obtain an overall assessment of $a^{i}$ : Construct an overall distance function for $a^{i}$ to aggregate the aforesaid two distances into a final evaluation result. The overall distance $D\left(a^{i}\right)$ can be expressed as [13]

$$
D\left(a^{i}\right)=\frac{D\left(a^{i}\right)^{-}}{D\left(a^{i}\right)^{-}+D\left(a^{i}\right)^{+}} .
$$

Obviously, a larger value of $D\left(a^{i}\right)$ represents a better overall performance.

\section{An OWA-TOPSIS method}

\subsection{Flexible settings of $a^{+}$and $a^{-}$}

As described in Section 2.3, the setting of ideal and anti-ideal points in TOPSIS is based upon value data, i.e. normalized and refined consequence data. According to the DM's 
preference directions over different criteria, $a^{+}$and $a^{-}$are set as the combinations of either maximum or minimum values of $v_{j}^{i}\left(\forall c_{j} \in \mathbf{C}\right.$ and $\left.\forall a^{i} \in \mathbf{A}\right)$. In practice, a DM often has ideal or anti-ideal alternatives (points) directly given as consequence data. For example, in business analysis various benchmarks have been proposed for company performance evaluations [1]. The current TOPSIS method does not provide a flexible mechanism to allow a DM to specify $a^{+}$and $a^{-}$in the consequence data space directly, but the DM may feel much easier and more meaningful compared to discussions in the value data space $[5,6]$.

To improve the setting flexibility, this article allows a DM to define $a^{+}$and $a^{-}$in the consequence data space directly as long as the following conditions are satisfied [5]:

(1) For a benefit criterion, $c_{g} \in \mathbf{C}$, and an alternative, $a^{i} \in \mathbf{A}, m_{g}\left(a^{+}\right) \geq m_{g}\left(a^{i}\right)$ and $m_{g}\left(a^{-}\right) \leq m_{g}\left(a^{i}\right)$.

(2) For a cost criterion, $c_{h} \in \mathbf{C}$, and an alternative, $a^{i} \in \mathbf{A}, m_{h}\left(a^{+}\right) \leq m_{h}\left(a^{i}\right)$ and $m_{h}\left(a^{-}\right) \geq m_{h}\left(a^{i}\right)$.

It is trivial to verify that the setting of $a^{+}$and $a^{-}$in the original TOPSIS method satisfies the above conditions. Hence, our new setting of $a^{+}$and $a^{-}$can be regarded as a logical extension of that in the original TOPSIS method.

Additionally, in the original TOPSIS method, a DM only sets one pair of extreme points, $a^{+}$and $a^{-}$. As an interesting extension, multiple extreme points may occur in a TOPSIS analysis, where a DM provides more than one pair of extreme points over different criteria or as a group decision support procedure, various extreme points suggested by different participants must be considered. Formally, let $\mathbf{O}=\left\{\left(o_{+}^{1}, o_{-}^{1}\right), \cdots,\left(o_{+}^{|\mathbf{O}|}, o_{-}^{|\mathbf{O}|}\right)\right\}$ be a set of ideal and anti-ideal point pairs. Furthermore, let $\mathbf{O}_{+}=\left\{o_{+}^{1}, \cdots, o_{+}^{|\mathbf{O}|}\right\}$ and $\mathbf{O}_{-}=\left\{o_{-}^{1}, \cdots, o_{-}^{|\mathbf{O}|}\right\}$ be a set of ideal points and anti-ideal points, respectively. Similarly, the setting of $\mathbf{O}_{+}$and $\mathbf{O}_{-}$over the criterion set $\mathbf{C}$ satisfies the following conditions:

(1) For a benefit criterion, $c_{g} \in \mathbf{C}$, and an alternative, $a^{i} \in \mathbf{A}, m_{g}\left(o_{+}^{k}\right) \geq m_{g}\left(a^{i}\right)$ and $m_{g}\left(o_{-}^{k}\right) \leq m_{g}\left(a^{i}\right)$, where $o_{+}^{k} \in \mathbf{O}_{+}$and $o_{-}^{k} \in \mathbf{O}_{-}$.

(2) For a cost criterion, $c_{h} \in \mathbf{C}$, and an alternative, $a^{i} \in \mathbf{A}, m_{h}\left(o_{+}^{k}\right) \leq m_{h}\left(a^{i}\right)$ and $m_{h}\left(o_{-}^{k}\right) \geq m_{h}\left(a^{i}\right)$, where $o_{+}^{k} \in \mathbf{O}_{+}$and $o_{-}^{k} \in \mathbf{O}_{-}$.

\subsection{Distance aggregations}

For each $c_{j} \in \mathbf{C}$, in the consequence space the distances from $a^{i}$ to an ideal point, $o_{+}^{k} \in$ $\mathbf{O}_{+}$and an anti-ideal point, $o_{-}^{l} \in \mathbf{O}_{-}$are measured by $m_{j}\left(o_{+}^{k}\right)-m_{j}\left(a^{i}\right)$ and $m_{j}\left(a^{i}\right)-$ $m_{j}\left(o_{-}^{l}\right)$, respectively. Then, any aforementioned normalization function in Section 2.3 can be utilized to obtain the normalized distances of $a^{i}$ to $o_{+}^{k}$ and $o_{-}^{l}$ in the value space, denoted as $d_{j}\left(a^{i}\right)_{+}^{k}$ and $d_{j}\left(a^{i}\right)_{-}^{l}$, respectively. The detailed normalization process is omitted here. 
To facilitate further discussions, the following notation is introduced:

- Let $d_{j}\left(a^{i}\right)_{+}$and $d_{j}\left(a^{i}\right)_{-}$be the aggregated distances between $a^{i}$ and the ideal and anti-ideal points set, $\mathbf{O}_{+}$and $\mathbf{O}_{-}$, over the criterion $c_{j}$, respectively.

- let $D\left(a^{i}\right)_{+}^{k}$ and $D\left(a^{i}\right)_{-}^{l}$ be the aggregated distances between $a^{i}$ and $o_{+}^{k}$ and $o_{-}^{l}$ over the criterion set $\mathbf{C}$, respectively. The values of $D\left(a^{i}\right)_{+}^{k}$ and $D\left(a^{i}\right)_{-}^{l}$ can be obtained by Eqs. (1) and (2) where the $p$-norm distance function needs to be pre-defined by a DM.

- Let $D\left(a^{i}\right)_{+}$and $D\left(a^{i}\right)_{-}$represent the overall distances of $a^{i}$ to $\mathbf{O}_{+}$and $\mathbf{O}_{-}$, respectively.

- Let $D\left(a^{i}\right)^{k}$ be the overall performance distance between $a^{i}$ and a pair of ideal and anti-ideal points, $\left(o_{+}^{k}, o_{-}^{k}\right)$, and let $D\left(a^{i}\right)$ be the overall performance distance of $a^{i}$ to O. The calculation of $D\left(a^{i}\right)^{k}$ or $D\left(a^{i}\right)$ is determined by Eq. (3).

To obtain the final distance performance of $a^{i}, D\left(a^{i}\right)$, beginning with $d_{j}\left(a^{i}\right)_{+}^{k}$ and $d_{j}\left(a^{i}\right)_{-}^{l}$, two kinds of information aggregation are needed:

- Preference aggregation over the criterion set $\mathbf{C}$ : Distances of an alternative over different criteria have to be aggregated based on the preferences over criteria or weights w. This type of information aggregation is commonly studied in MCDA and, hence, is denoted as $\mathrm{MCDA}_{\mathbf{w}}$.

- Source aggregation over the ideal and anti-ideal point sets, $\mathbf{O}_{+}$and $\mathbf{O}_{-}$: Similarly, different distance information between an alternative and various ideal or anti-ideal points within $\mathbf{O}_{+}$and $\mathbf{O}_{-}$, has to be aggregated to reflect different opinions from distinct ideal and anti-ideal points of view. Here, the OWA aggregation associated with a set of weights, $\mathbf{Q}$, is used to achieve this operation. This type of aggregation is denoted as $\mathrm{OWA}_{\mathbf{Q}}$.

Next, three aggregation scenarios that integrate OWA into TOPSIS are designed as follows:

- Internal aggregation: The overall information process of an internal aggregation is shown below:

$$
\left.\begin{array}{l}
\left\{d_{j}\left(a^{i}\right)_{+}^{k}\right\} \stackrel{O W A_{\mathbf{Q}}}{\longrightarrow}\left\{d_{j}\left(a^{i}\right)_{+}\right\} \stackrel{M C D A_{\mathbf{w}}}{\longrightarrow}\left\{D\left(a^{i}\right)_{+}\right\} \\
\left\{d_{j}\left(a^{i}\right)_{-}^{k}\right\} \stackrel{O W A_{\mathbf{Q}}}{\longrightarrow}\left\{d_{j}\left(a^{i}\right)_{-}\right\} \stackrel{M C D A_{\mathbf{w}}}{\longrightarrow}\left\{D\left(a^{i}\right)_{-}\right\}
\end{array}\right\} D\left(a^{i}\right)
$$

First, the distances between $a^{i}$ and different ideal and anti-ideal points, $o_{+}^{k}$ and $o_{-}^{k}$ over a criterion $c_{j}, \forall c_{j} \in \mathbf{C}$, are aggregated through $O W A_{\mathbf{P}}$; then the generated $d_{j}\left(a^{i}\right)_{+}$and $d_{j}\left(a^{i}\right)_{-}$are aggregated through $M C D A_{\mathbf{w}}$ over the criterion set $\mathbf{C}$ to produce $D\left(a^{i}\right)_{+}$and $D\left(a^{i}\right)_{-}$; finally, $D\left(a^{i}\right)$ is calculated using the aforementioned TOPSIS distance aggregation procedure in Eq. (3).

- External aggregation I: The overall information process of an external aggregation I is shown below:

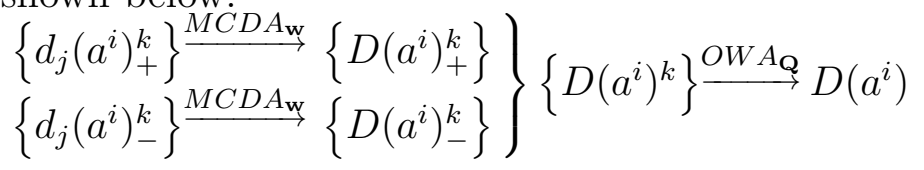


First, the distances between $a^{i}$ and the pair of $\left(o_{+}^{k}, o_{-}^{k}\right)$ over the criterion set $\mathbf{C}$ are aggregated through $M C D A_{\mathbf{w}}$; then the generated $D\left(a^{i}\right)_{+}^{k}$ and $D\left(a^{i}\right)_{-}^{k}$ are aggregated through Eq. (3) to produce $D\left(a^{i}\right)^{k}$; finally, $D\left(a^{i}\right)$ is obtained through $O W A_{\mathbf{Q}}$.

- External aggregation II: The general information process of an external aggregation II is shown below:

$$
\left.\begin{array}{l}
\left\{d_{j}\left(a^{i}\right)_{+}^{k}\right\} \stackrel{M C D \dot{A}_{\mathbf{w}}}{\longrightarrow}\left\{D\left(a^{i}\right)_{+}^{k}\right\} \stackrel{O W A_{\mathbf{Q}}}{\longrightarrow}\left\{D\left(a^{i}\right)_{+}\right\} \\
\left\{d_{j}\left(a^{i}\right)_{-}^{k}\right\} \stackrel{M C D A_{\mathrm{w}}}{\longrightarrow}\left\{D\left(a^{i}\right)_{-}^{k}\right\} \stackrel{O W A_{\mathbf{Q}}}{\longrightarrow}\left\{D\left(a^{i}\right)_{-}\right\}
\end{array}\right\} D\left(a^{i}\right)
$$

First, the distances between $a^{i}$ and the pair of $\left(o_{+}^{k}, o_{-}^{k}\right)$ over the criterion set $\mathbf{C}$ are aggregated through $M C D A_{\mathbf{w}}$; then the generated $D\left(a^{i}\right)_{+}^{k}$ and $D\left(a^{i}\right)_{-}^{k}$ are aggregated through $O W A_{\mathbf{Q}}$ to produce $D\left(a^{i}\right)_{+}$and $D\left(a^{i}\right)_{-}$; finally, $D\left(a^{i}\right)$ is obtained using Eq. (3).

The difference between the internal aggregation and the two external aggregations is the order of applying $M C D A_{\mathbf{w}}$ and $O W A_{\mathbf{Q}}$. In the internal aggregation, the $O W A_{\mathbf{Q}}$ is applied first to aggregate distances from different sources (both ideal or anti-ideal points), then $M C D A_{\mathrm{w}}$ is used to aggregate different information over various criteria. Such an information processing approach is appropriate for a single DM with multiple pairs of extreme points.

In the two external aggregations, $M C D A_{\mathbf{w}}$ is entertained first to aggregate different information over all criteria for each pair of extreme points. Different weight sets may be used in $M C D A_{\mathbf{w}}$ aggregations. Then, $O W A_{\mathbf{Q}}$ is utilized to aggregate the obtained information from different pairs of extreme points. These information processing schemes can be applied to a group decision support procedure where multiple participants provide different pairs of extreme points and different weight information. A case study adapted from [23] in which Shih et al. extended the TOPSIS method for group decision making, is explored in the next section to demonstrate the proposed method and compare the results from the above three aggregation scenarios.

\section{A numerical example}

\subsection{Background information}

A local chemical company is recruiting an on-site business manager [23]. 17 qualified candidates (alternatives) (labelled as $a^{1}-a^{17}$ ) compete for this position, and four decision makers (labelled as $D M_{1}-D M_{4}$ ) constitute the search committee. The human resources department provides some relevant selection tests: three knowledge tests covering language, professional and safety rules knowledge and two skill tests evaluating professional and computer skills, as well as two interviews involving panel interview with the search committee and 1-on-1 interview with individual committee members. These tests and interviews form the criterion set $\mathbf{C}$ and are labelled as $c_{1}-c_{7}$ sequentially according to 
the aforesaid order.

Obviously, the first five criteria, $c_{1}$ to $c_{5}$, provide relative objective measurements, while $c_{6}$ and $c_{7}$ rely on the DMs' subjective judgements for each candidate or alternative. The basic consequence information of these 17 alternatives over the seven criteria is listed in Table 1. Note that DMs differ in their subjective judgements for the alternatives according to the last two criteria, $c_{6}$ and $c_{7}$. Also, DMs have different settings of the relative importance of criteria which are shown in Table 2.

Table 1

Basic information [23]

\begin{tabular}{l|ccccc|cc|cc|cc|cc}
\hline \multirow{2}{*}{ Alternative } & \multicolumn{6}{|c|}{ Criteria } & \multicolumn{2}{|c|}{$D M_{1}$} & \multicolumn{2}{c|}{$D M_{2}$} & \multicolumn{2}{|c|}{$D M_{3}$} & \multicolumn{2}{|c}{$D M_{4}$} \\
& $c_{1}$ & $c_{2}$ & $c_{3}$ & $c_{4}$ & $c_{5}$ & $c_{6}$ & $c_{7}$ & $c_{6}$ & $c_{7}$ & $c_{6}$ & $c_{7}$ & $c_{6}$ & $c_{7}$ \\
\hline$a^{1}$ & 80 & 70 & 87 & 77 & 76 & 80 & 75 & 85 & 80 & 75 & 70 & 90 & 85 \\
$a^{2}$ & 85 & 65 & 76 & 80 & 75 & 65 & 75 & 60 & 70 & 70 & 77 & 60 & 70 \\
$a^{3}$ & 78 & 90 & 72 & 80 & 85 & 90 & 85 & 80 & 85 & 80 & 90 & 90 & 95 \\
$a^{4}$ & 75 & 84 & 69 & 85 & 65 & 65 & 70 & 55 & 60 & 68 & 72 & 62 & 72 \\
$a^{5}$ & 84 & 67 & 60 & 75 & 85 & 75 & 80 & 75 & 80 & 50 & 55 & 70 & 75 \\
$a^{6}$ & 85 & 78 & 82 & 81 & 79 & 80 & 80 & 75 & 85 & 77 & 82 & 75 & 75 \\
$a^{7}$ & 77 & 83 & 74 & 70 & 71 & 65 & 70 & 70 & 60 & 65 & 72 & 67 & 75 \\
$a^{8}$ & 78 & 82 & 72 & 80 & 78 & 70 & 60 & 75 & 65 & 75 & 67 & 82 & 85 \\
$a^{9}$ & 85 & 90 & 80 & 88 & 90 & 80 & 85 & 95 & 85 & 90 & 85 & 90 & 92 \\
$a^{10}$ & 89 & 75 & 79 & 67 & 77 & 70 & 75 & 75 & 80 & 68 & 78 & 65 & 70 \\
$a^{11}$ & 65 & 55 & 68 & 62 & 70 & 50 & 60 & 62 & 65 & 60 & 65 & 65 & 70 \\
$a^{12}$ & 70 & 64 & 65 & 65 & 60 & 60 & 65 & 65 & 75 & 50 & 60 & 45 & 50 \\
$a^{13}$ & 95 & 80 & 70 & 75 & 70 & 75 & 75 & 80 & 80 & 65 & 75 & 70 & 75 \\
$a^{14}$ & 70 & 80 & 79 & 80 & 85 & 80 & 70 & 75 & 72 & 80 & 70 & 75 & 75 \\
$a^{15}$ & 60 & 78 & 87 & 70 & 66 & 70 & 65 & 75 & 70 & 65 & 70 & 60 & 65 \\
$a^{16}$ & 92 & 85 & 88 & 90 & 85 & 90 & 95 & 92 & 90 & 85 & 80 & 88 & 90 \\
$a^{17}$ & 86 & 87 & 80 & 70 & 72 & 80 & 85 & 70 & 75 & 75 & 80 & 70 & 75 \\
\hline
\end{tabular}

Table 2

Weight information from DMs [23]

\begin{tabular}{c|ccccccc}
\hline & $c_{1}$ & $c_{2}$ & $c_{3}$ & $c_{4}$ & $c_{5}$ & $c_{6}$ & $c_{7}$ \\
\hline$D M_{1}$ & 0.066 & 0.196 & 0.066 & 0.130 & 0.130 & 0.216 & 0.196 \\
$D M_{2}$ & 0.042 & 0.112 & 0.082 & 0.176 & 0.118 & 0.215 & 0.255 \\
$D M_{3}$ & 0.060 & 0.134 & 0.051 & 0.167 & 0.100 & 0.203 & 0.285 \\
$D M_{4}$ & 0.047 & 0.109 & 0.037 & 0.133 & 0.081 & 0.267 & 0.326 \\
\hline
\end{tabular}

\subsection{The setting of ideal and anti-ideal sets}

Here, the same preference assumptions in Shih et al. [23] are employed for conducting an OWA-TOPSIS analysis. The details are given below: 
- All criteria are benefit and, hence, preference monotonic.

- The four pairs of extreme points provided by the four DMs constitute $\mathbf{O}$. The settings of $o_{+}^{k}$ and $o_{-}^{k}$ follow the rule that $\forall c_{j} \in \mathbf{C}, m_{j}\left(o_{+}^{k}\right)=\max _{i=1}^{17} m_{j}\left(a^{i}\right)$, and $m_{j}\left(o_{-}^{k}\right)=\min _{i=1}^{17} m_{j}\left(a^{i}\right)$, and are shown in Table 3. Note that there is no explicit account for multiple pairs of extreme points in [23], although the four DMs have different extreme point settings regarding $c_{6}$ and $c_{7}$. As an extension of Shih et al.'s method, the proposed OWA-TOPSIS method provides a general framework of information aggregation regarding multiple pairs of extreme points and multiple criteria.

- The vector normalization is used to normalize the data.

Table 3

Ideal and anti-ideal points setting for DMs [23]

\begin{tabular}{c|c|ccccccc}
\hline DMs & $\left(o_{+}^{k}, o_{-}^{k}\right)$ & $c_{1}$ & $c_{2}$ & $c_{3}$ & $c_{4}$ & $c_{5}$ & $c_{6}$ & $c_{7}$ \\
\hline \multirow{2}{*}{$D M_{1}$} & $o_{+}^{1}$ & 95 & 90 & 88 & 90 & 90 & 90 & 95 \\
& $o_{-}^{1}$ & 60 & 55 & 60 & 62 & 60 & 50 & 60 \\
\hline \multirow{2}{*}{$D M_{2}$} & $o_{+}^{2}$ & 95 & 90 & 88 & 90 & 90 & 95 & 90 \\
& $o_{-}^{2}$ & 60 & 55 & 60 & 62 & 60 & 55 & 60 \\
\hline \multirow{2}{*}{$D M_{3}$} & $o_{+}^{3}$ & 95 & 90 & 88 & 90 & 90 & 90 & 90 \\
& $o_{-}^{3}$ & 60 & 55 & 60 & 62 & 60 & 50 & 55 \\
\hline \multirow{2}{*}{$D M_{4}$} & $o_{+}^{4}$ & 95 & 90 & 88 & 90 & 90 & 90 & 95 \\
& $o_{-}^{4}$ & 60 & 55 & 60 & 62 & 60 & 45 & 50 \\
\hline
\end{tabular}

\subsection{The setting of $O W A_{\mathbf{Q}}$}

The fuzzy linguistic quantifiers approach [28] to determining the ordered weights provides various information regarding decision behaviors. Let $q_{i}=\left(\frac{i}{|\mathbf{B}|}\right)^{\alpha}-\left(\frac{i-1}{|\mathbf{B}|}\right)^{\alpha}$, for $i=1, \ldots,|\mathbf{B}|$, where $\alpha$ indicates the degree of inclusion for different elements. By changing the $\alpha$ value, different decision strategies are induced [17]. Table 4 demonstrates weight settings for different fuzzy linguistic quantifiers under $|\mathbf{B}|=4$. For example, $\alpha=0$ (at least one) indicates that an DM will get absolutely no satisfaction if there is no satisfaction for the DM from any of four pieces of information, i.e. this setting of $\alpha$ achieves the "OR" operation. Subsequently, $\alpha=0.1,0.5,1,2,10,1000$ represent the linguistic words of "few", "some", "half (average)", "many", "most", and "all", respectively.

\subsection{Internal and external aggregations}

It is assumed that the distance norm, $p$, is set at $p=1$ and the weight information in Table 2 is used for $M C D A_{\mathrm{w}}$ aggregation. The weight information in Table 4 is applied for $O W A_{\mathbf{Q}}$ aggregation. Then, the aforementioned three aggregation processes, internal 
Table 4

Order Weights for Selected Linguistic Quantifiers [28]

\begin{tabular}{c|c|c|c|c|c|c|c}
\hline Linguistic quantifier & At least one & Few & Some & Half (Average) & Many & Most & All \\
$\alpha$ & 0 & 0.1 & 0.5 & 1 & 2 & 10 & 1000 \\
\hline$q_{1}$ & 1.0000 & 0.8706 & 0.5000 & 0.2500 & 0.0625 & 0.0000 & 0.0000 \\
$q_{2}$ & 0.0000 & 0.0625 & 0.2071 & 0.2500 & 0.1875 & 0.0010 & 0.0000 \\
$q_{3}$ & 0.0000 & 0.0386 & 0.1589 & 0.2500 & 0.3125 & 0.0553 & 0.0000 \\
$q_{4}$ & 0.0000 & 0.0284 & 0.1340 & 0.2500 & 0.4375 & 0.9437 & 1.0000 \\
\hline
\end{tabular}

aggregation, external aggregation I, and external aggregation II as discussed in Section 3.2, are conducted and the results are shown in Tables 5, 6 and 7, respectively, where R1-R7 refer to different alternative rankings based on the seven predefined $\alpha$ values in Table 4. Note that in the internal aggregation (one DM with multiple pairs of extreme points) as shown in Table 5, the weight information from $D M_{1},(0.0660,0.1960,0.0660$, $0.1300,0.1300,0.2160,0.1960)$ in Table 4 is used for $M C D A_{\mathbf{w}}$ aggregation, and in the two external aggregations (multiple DMs with multiple pairs of extreme points) as given in Tables 6 and 7, different criterion weight information shown in Table 2 is employed for $M C D A_{\mathbf{w}}$ aggregations.

Table 5

Final distance performance and rankings of alternative in internal aggregation

\begin{tabular}{c|cc|cc|cc|cc|cc|cc|cc}
\hline \multirow{2}{*}{$\mathbf{A}$} & \multicolumn{2}{|c|}{$\alpha=0$} & \multicolumn{2}{c|}{$\alpha=0.1$} & \multicolumn{2}{c|}{$\alpha=0.5$} & \multicolumn{2}{c|}{$\alpha=1$} & \multicolumn{2}{c|}{$\alpha=2$} & \multicolumn{2}{c|}{$\alpha=10$} & \multicolumn{2}{c}{$\alpha=1000$} \\
& $D\left(a^{i}\right)$ & $\mathrm{R} 1$ & $D\left(a^{i}\right)$ & $\mathrm{R} 2$ & $D\left(a^{i}\right)$ & $\mathrm{R} 3$ & $D\left(a^{i}\right)$ & $\mathrm{R} 4$ & $D\left(a^{i}\right)$ & $\mathrm{R} 5$ & $D\left(a^{i}\right)$ & $\mathrm{R} 6$ & $D\left(a^{i}\right)$ & $\mathrm{R} 7$ \\
\hline$a^{1}$ & 0.5620 & 7 & 0.6032 & 5 & 0.5687 & 7 & 0.5740 & 7 & 0.5817 & 7 & 0.6001 & 5 & 0.6022 & 5 \\
$a^{2}$ & 0.4346 & 11 & 0.4292 & 12 & 0.4247 & 13 & 0.4191 & 14 & 0.4144 & 14 & 0.4114 & 11 & 0.4113 & 11 \\
$a^{3}$ & 0.7434 & 3 & 0.7549 & 3 & 0.7716 & 3 & 0.7908 & 3 & 0.8123 & 3 & 0.8299 & 3 & 0.8297 & 3 \\
$a^{4}$ & 0.4257 & 12 & 0.4211 & 14 & 0.4333 & 11 & 0.4342 & 11 & 0.4276 & 11 & 0.3902 & 13 & 0.3855 & 13 \\
$a^{5}$ & 0.4011 & 14 & 0.4962 & 11 & 0.4208 & 14 & 0.4275 & 12 & 0.4214 & 13 & 0.3553 & 15 & 0.3462 & 15 \\
$a^{6}$ & 0.6192 & 4 & 0.6584 & 4 & 0.6338 & 4 & 0.6425 & 4 & 0.6510 & 4 & 0.6576 & 4 & 0.6578 & 4 \\
$a^{7}$ & 0.4207 & 13 & 0.4247 & 13 & 0.4257 & 12 & 0.4263 & 13 & 0.4223 & 12 & 0.4019 & 12 & 0.3996 & 12 \\
$a^{8}$ & 0.5469 & 8 & 0.5553 & 9 & 0.5408 & 8 & 0.5396 & 8 & 0.5439 & 8 & 0.5730 & 8 & 0.5769 & 8 \\
$a^{9}$ & 0.8457 & 2 & 0.9146 & 2 & 0.8788 & 2 & 0.8975 & 2 & 0.9134 & 2 & 0.9240 & 2 & 0.9246 & 2 \\
$a^{10}$ & 0.4799 & 10 & 0.5213 & 10 & 0.4839 & 10 & 0.4857 & 10 & 0.4867 & 10 & 0.4851 & 10 & 0.4849 & 10 \\
$a^{11}$ & 0.1903 & 16 & 0.1961 & 17 & 0.1647 & 16 & 0.1459 & 16 & 0.1219 & 16 & 0.0796 & 17 & 0.0757 & 17 \\
$a^{12}$ & 0.1900 & 17 & 0.2193 & 16 & 0.1616 & 17 & 0.1423 & 17 & 0.1208 & 17 & 0.0976 & 16 & 0.0964 & 16 \\
$a^{13}$ & 0.5268 & 9 & 0.5741 & 7 & 0.5277 & 9 & 0.5286 & 9 & 0.5299 & 9 & 0.5328 & 9 & 0.5330 & 9 \\
$a^{14}$ & 0.5842 & 5 & 0.5876 & 6 & 0.5885 & 5 & 0.5910 & 5 & 0.5934 & 5 & 0.5954 & 6 & 0.5954 & 6 \\
$a^{15}$ & 0.3861 & 15 & 0.4127 & 15 & 0.3799 & 15 & 0.3756 & 15 & 0.3702 & 15 & 0.3589 & 14 & 0.3576 & 14 \\
$a^{16}$ & 0.8605 & 1 & 0.9208 & 1 & 0.8842 & 1 & 0.8990 & 1 & 0.9143 & 1 & 0.9283 & 1 & 0.9288 & 1 \\
$a^{17}$ & 0.5748 & 6 & 0.5728 & 8 & 0.5790 & 6 & 0.5824 & 6 & 0.5870 & 6 & 0.5943 & 7 & 0.5947 & 7 \\
\hline
\end{tabular}


Table 6

Final distance performance and rankings of alternatives in external aggregation I

\begin{tabular}{c|ccc|cc|cc|cc|cc|cc|cc}
\hline \multirow{2}{*}{$\mathbf{A}$} & \multicolumn{2}{|c|}{$\alpha=0$} & \multicolumn{2}{c|}{$\alpha=0.1$} & \multicolumn{2}{c|}{$\alpha=0.5$} & \multicolumn{2}{c|}{$\alpha=1$} & \multicolumn{2}{c|}{$\alpha=2$} & \multicolumn{2}{c|}{$\alpha=10$} & \multicolumn{2}{c}{$\alpha=1000$} \\
& $D\left(a^{i}\right)$ & $\mathrm{R} 1$ & $D\left(a^{i}\right)$ & $\mathrm{R} 2$ & $D\left(a^{i}\right)$ & $\mathrm{R} 3$ & $D\left(a^{i}\right)$ & $\mathrm{R} 4$ & $D\left(a^{i}\right)$ & $\mathrm{R} 5$ & $D\left(a^{i}\right)$ & $\mathrm{R} 6$ & $D\left(a^{i}\right)$ & $\mathrm{R} 7$ \\
\hline$a^{1}$ & 0.7059 & 4 & 0.6889 & 4 & 0.6365 & 5 & 0.5951 & 5 & 0.5528 & 6 & 0.5089 & 6 & 0.5068 & 6 \\
$a^{2}$ & 0.5227 & 10 & 0.5077 & 11 & 0.4635 & 12 & 0.4311 & 11 & 0.4017 & 11 & 0.3777 & 11 & 0.3765 & 11 \\
$a^{3}$ & 0.8814 & 3 & 0.8684 & 3 & 0.8291 & 3 & 0.7987 & 3 & 0.7673 & 3 & 0.7201 & 3 & 0.7156 & 3 \\
$a^{4}$ & 0.5118 & 12 & 0.4994 & 12 & 0.4597 & 13 & 0.4252 & 13 & 0.3822 & 12 & 0.2936 & 14 & 0.2843 & 14 \\
$a^{5}$ & 0.5078 & 13 & 0.4983 & 13 & 0.4642 & 11 & 0.4287 & 12 & 0.3747 & 14 & 0.2415 & 15 & 0.2274 & 15 \\
$a^{6}$ & 0.6831 & 6 & 0.6781 & 5 & 0.6616 & 4 & 0.6466 & 4 & 0.6274 & 4 & 0.5899 & 4 & 0.5863 & 4 \\
$a^{7}$ & 0.4584 & 14 & 0.4511 & 14 & 0.4271 & 14 & 0.4050 & 14 & 0.3759 & 13 & 0.3159 & 12 & 0.3099 & 13 \\
$a^{8}$ & 0.6922 & 5 & 0.6660 & 6 & 0.5887 & 7 & 0.5330 & 8 & 0.4846 & 9 & 0.4550 & 9 & 0.4545 & 9 \\
$a^{9}$ & 0.9344 & 2 & 0.9286 & 2 & 0.9103 & 2 & 0.8945 & 2 & 0.8751 & 2 & 0.8358 & 2 & 0.8317 & 2 \\
$a^{10}$ & 0.5141 & 11 & 0.5088 & 10 & 0.4912 & 10 & 0.4749 & 10 & 0.4536 & 10 & 0.4136 & 10 & 0.4099 & 10 \\
$a^{11}$ & 0.2667 & 16 & 0.2504 & 16 & 0.2008 & 16 & 0.1621 & 16 & 0.1220 & 16 & 0.0686 & 16 & 0.0642 & 16 \\
$a^{12}$ & 0.2432 & 17 & 0.2273 & 17 & 0.1784 & 17 & 0.1401 & 17 & 0.1007 & 17 & 0.0541 & 17 & 0.0509 & 17 \\
$a^{13}$ & 0.5614 & 9 & 0.5554 & 9 & 0.5374 & 9 & 0.5237 & 9 & 0.5108 & 8 & 0.4999 & 7 & 0.4994 & 7 \\
$a^{14}$ & 0.5903 & 8 & 0.5887 & 8 & 0.5832 & 8 & 0.5779 & 6 & 0.5706 & 5 & 0.5555 & 5 & 0.5540 & 5 \\
$a^{15}$ & 0.4035 & 15 & 0.3977 & 15 & 0.3796 & 15 & 0.3643 & 15 & 0.3464 & 15 & 0.3158 & 13 & 0.3130 & 12 \\
$a^{16}$ & 0.9391 & 1 & 0.9346 & 1 & 0.9189 & 1 & 0.9036 & 1 & 0.8825 & 1 & 0.8418 & 1 & 0.8382 & 1 \\
$a^{17}$ & 0.6465 & 7 & 0.6338 & 7 & 0.5936 & 6 & 0.5603 & 7 & 0.5239 & 7 & 0.4840 & 8 & 0.4822 & 8 \\
\hline
\end{tabular}

Table 7

Final distance performance and rankings of alternatives in external aggregation II

\begin{tabular}{c|ccc|cc|cc|cc|cc|cc|cc}
\hline \multirow{2}{*}{$\mathbf{A}$} & \multicolumn{2}{|c|}{$\alpha=0$} & \multicolumn{2}{c|}{$\alpha=0.1$} & \multicolumn{2}{c|}{$\alpha=0.5$} & \multicolumn{2}{c|}{$\alpha=1$} & \multicolumn{2}{c|}{$\alpha=2$} & \multicolumn{2}{c|}{$\alpha=10$} & \multicolumn{2}{c}{$\alpha=1000$} \\
& $D\left(a^{i}\right)$ & $\mathrm{R} 1$ & $D\left(a^{i}\right)$ & $\mathrm{R} 2$ & $D\left(a^{i}\right)$ & $\mathrm{R} 3$ & $D\left(a^{i}\right)$ & $\mathrm{R} 4$ & $D\left(a^{i}\right)$ & $\mathrm{R} 5$ & $D\left(a^{i}\right)$ & $\mathrm{R} 6$ & $D\left(a^{i}\right)$ & $\mathrm{R} 7$ \\
\hline$a^{1}$ & 0.5769 & 5 & 0.5787 & 5 & 0.7647 & 5 & 0.5939 & 5 & 0.6071 & 5 & 0.6405 & 4 & 0.6440 & 4 \\
$a^{2}$ & 0.4584 & 11 & 0.4537 & 11 & 0.6213 & 13 & 0.4310 & 11 & 0.4261 & 11 & 0.4368 & 11 & 0.4385 & 11 \\
$a^{3}$ & 0.7452 & 3 & 0.7526 & 3 & 0.9013 & 3 & 0.7975 & 3 & 0.8207 & 3 & 0.8604 & 3 & 0.8647 & 3 \\
$a^{4}$ & 0.4191 & 12 & 0.4210 & 12 & 0.6329 & 12 & 0.4256 & 13 & 0.4174 & 13 & 0.3719 & 12 & 0.3659 & 12 \\
$a^{5}$ & 0.3917 & 14 & 0.3986 & 14 & 0.6468 & 11 & 0.4279 & 12 & 0.4211 & 12 & 0.3330 & 15 & 0.3203 & 15 \\
$a^{6}$ & 0.6831 & 4 & 0.6762 & 4 & 0.7807 & 4 & 0.6466 & 4 & 0.6405 & 4 & 0.6395 & 5 & 0.6389 & 5 \\
$a^{7}$ & 0.4021 & 13 & 0.4034 & 13 & 0.6020 & 14 & 0.4052 & 14 & 0.3979 & 14 & 0.3650 & 13 & 0.3611 & 13 \\
$a^{8}$ & 0.5560 & 7 & 0.5513 & 8 & 0.7179 & 8 & 0.5322 & 8 & 0.5364 & 8 & 0.5908 & 6 & 0.5984 & 6 \\
$a^{9}$ & 0.8446 & 2 & 0.8523 & 2 & 0.9542 & 2 & 0.8941 & 2 & 0.9110 & 2 & 0.9282 & 2 & 0.9297 & 2 \\
$a^{10}$ & 0.4593 & 10 & 0.4621 & 10 & 0.6600 & 10 & 0.4745 & 10 & 0.4759 & 10 & 0.4653 & 10 & 0.4639 & 10 \\
$a^{11}$ & 0.2205 & 16 & 0.2128 & 16 & 0.3322 & 16 & 0.1620 & 16 & 0.1324 & 16 & 0.0854 & 16 & 0.0811 & 16 \\
$a^{12}$ & 0.1912 & 17 & 0.1843 & 17 & 0.2968 & 17 & 0.1381 & 17 & 0.1107 & 17 & 0.0710 & 17 & 0.0680 & 17 \\
$a^{13}$ & 0.5279 & 9 & 0.5269 & 9 & 0.6947 & 9 & 0.5237 & 9 & 0.5250 & 9 & 0.5324 & 9 & 0.5331 & 9 \\
$a^{14}$ & 0.5706 & 6 & 0.5718 & 6 & 0.7377 & 6 & 0.5779 & 6 & 0.5797 & 6 & 0.5789 & 7 & 0.5787 & 7 \\
$a^{15}$ & 0.3603 & 15 & 0.3612 & 15 & 0.5511 & 15 & 0.3638 & 15 & 0.3620 & 15 & 0.3543 & 14 & 0.3537 & 14 \\
$a^{16}$ & 0.8533 & 1 & 0.8609 & 1 & 0.9601 & 1 & 0.9036 & 1 & 0.9211 & 1 & 0.9320 & 1 & 0.9321 & 1 \\
$a^{17}$ & 0.5543 & 8 & 0.5548 & 7 & 0.7359 & 7 & 0.5601 & 7 & 0.5655 & 7 & 0.5770 & 8 & 0.5779 & 8 \\
\hline
\end{tabular}




\subsection{Result comparison}

Tables 5, 6, and 7 clearly indicate the 21 rankings resulted from the three aggregation scenarios under the seven $\alpha$ settings unanimously identify the first three candidates: $a^{16}$, $a^{9}$, and $a^{3}$. For remaining candidates, rankings are slightly different. Next, Friedman tests [12] are carried out to detect ordinal ranking differences under different $\alpha$ settings of OWA and internal and external aggregation schemes. The Friedman test is a nonparametric statistical test that is developed by a Nobel Laureate economist, Milton Friedman. A classic example is that given the rankings (or ratings) of $k$ different wines by $n$ wine judges, the Friedman test can assess whether the judgements are consistent from a statistical point of view [12]. Therefore, it is appropriate to employ this technique to investigate the consistency of rankings under different settings as shown in Tables 5 , 6 , and 7 .

In the case of internal aggregation, the hypotheses is:

- $H_{0}$ : The 7 rankings $(\alpha=0,0.1,0.5,1,2,10,1000)$ of 17 alternatives are the same.

- $H_{1}$ : At least two rankings are different.

The test result listed in Table 8 indicates that one should not reject $H_{0}$ in favour of $H_{1}$ at the $5 \%$ significance level. In the context of this test, there is no sufficient statistical evidence to infer that the 7 ranking results are different. In other words, although the 7 approaches based on various $\alpha$ values generate slightly different ranking orders, the Friedman test shows that the rankings are not significantly different and there exists overall consistency in the rankings based on the seven $\alpha$ settings for internal aggregation.

Table 8

Friedman Test Result I

\begin{tabular}{c|c}
\hline$Q$ (observed value) & 1.205 \\
\hline$Q$ (critical value) & 12.592 \\
\hline $\mathrm{DF}$ & 6 \\
\hline One-tailed $p$-value & 0.977 \\
\hline Alpha & 0.05 \\
\hline
\end{tabular}

Similarly, the Friedman test confirms that the total 14 different $\alpha$ settings for external aggregation I and II (each aggregation contains 7 settings of $\alpha$ ) also produce overall consistent rankings of alternatives. The test result is shown in Table 9.

Table 9

Friedman Test Result II

\begin{tabular}{c|c}
\hline$Q$ (observed value) & 0.861 \\
\hline$Q$ (critical value) & 22.362 \\
\hline $\mathrm{DF}$ & 13 \\
\hline One-tailed $p$-value & 1.000 \\
\hline Alpha & 0.05 \\
\hline
\end{tabular}




\section{Conclusions}

In this paper, a hybrid approach is designed to integrate OWA operators into the TOPSIS analysis procedure to achieve diverse information aggregations for multiple criteria decision analysis. The proposed method can be regarded as an extension of the approach by Shih et al. [23] and information aggregations are categorized in two groups: preference and source aggregations. The preference aggregation is attained by aggregating information over different criteria; the source aggregation is fulfilled by OWA operators over different pairs of extreme points. Given different combinations of these two information aggregations, three scenarios (internal aggregation, external aggregation I, and external aggregation II) are devised to generate final rankings. A numerical example demonstrates the approach and comparative results confirm the statistical consistency of the ranking results by the three aggregation scenarios with different $\alpha$ settings.

\section{References}

[1] Brook-Hart, G. (2006). Business Benchmark. Cambridge, UK: Cambridge University Press, (Chapter 1).

[2] Chen, C. T. (2000). Extensions of the TOPSIS for group decision-making under fuzzy environment. Fuzzy Sets and Systems, 114, 1-9.

[3] Chen, M.F., \& Tzeng, G. H. (2004). Combining gray relation and TOPSIS concepts for selecting an expatriate host country. Mathematical and Computer Modelling, 40, 14731490 .

[4] Chen, Y., Li, K. W., Kilgour, D. M., \& Hipel, K. W. (2008). A case-based distance model for multiple criteria ABC analysis. Computers and Operations Research, 35, 776-796.

[5] Chen, Y., Kilgour, D. M., \& Hipel, K. W. (2009a). An extreme point-based distance approach to multiple criteria ranking. submitted to a journal for review.

[6] Chen, Y., Li, K. W., Xu, H., \& Liu, S. (2009b). A DEA-TOPSIS for multiple criteria ranking. submitted to a journal for review.

[7] Chen, Y., Kilgour, D. M., \& Hipel, K. W. (2006). Multiple criteria classification with an application in water resources planning. Computers and Operations Research, 33, 33013323 .

[8] Cheng, C., Wang, J., \& Wu, M. (2009). OWA-weighted based clustering method for classification problem. Expert Systems with Applications, 36, 4988-4995.

[9] Chu, T., \& Lin, Y. (2009). An interval arithmetic based fuzzy TOPSIS model. Expert Systems with Applications. 36, 10870-10876. 
[10] Department of the Environment, Transport and the Regions (DETR), UK, A New Deal for Trunk Roads in England: Understanding the New Approach to Appraisal. London, UK, 1998.

[11] Figueira, J., Greco, S., \& Ehrgott, M. (2005). (Eds.) Multiple Criteria Decision Analysis: State of the Art Surveys. New York: Springer.

[12] Friedman, M. (1937). The use of ranks to avoid the assumption of normality implicit in the analysis of variance. Journal of the American Statistical Association, 32, 675-701.

[13] Hwang, C. L. \& Yoon, K. (1981). Multiple Attribute Decision Making. Berlin: SpringerVerlag.

[14] Hwang, C. L., Lai, Y. J., \& Liu, T. Y. (1993). A new approach for multiple objective decision making. Computers and Operations Research, 20, 889-899.

[15] O'Hagan, M. (1988). Aggregating template rule antecedents in real-time expert systems with fuzzy set logic. In: Proceedings of the 22nd Annual IEEE Asilomar Conference on Signals, Systems, Computers, Pacific Grove, CA, US, 681-689.

[16] Lai, Y. J., Liu, T. Y, \& Hwang, C. L. (1994). TOPSIS for MODM. European Journal of Operational Research, 76, 486-500.

[17] Malczewski, J., \& Rinner, C. (2005). Exploring multicriteria decision strategies in GIS with linguistic quantifiers: a case study of residential quality evaluation. Journal of Geograph Systems, 7, 249-268.

[18] Keeney, R. L., \& Raiffa H. (1976). Decisions with Multiple Objectives: Preferences and Value Tradeoffs. New York: Wiley.

[19] Kim, G., Park, C.S., \& Yoon, K. P. (1997). Identifying investment opportunities for advanced manufacturing systems with comparative integrated performance measurement. International Journal of Production Economics, 50, 23-33.

[20] Olcer, A. I. (2008). A hybrid approach for multi-objective combinatorial optimisation problems in ship design and shipping. Computers and Operations Research, 35, 27602775 .

[21] Roy, B. (1996). Multicriteria Methodology for Decision Aiding. Dordrecht: Kluwer.

[22] Saaty, T. L. (1980). Analytic Hierarchy Process. New York: McGraw Hill.

[23] Shih, H. S., Shyur, H.J., \& Lee, E. S. (2007). An extension of TOPSIS for group decision making. Mathematical and Computer Modelling, 45, 801-813.

[24] Torra, V., \& Narukawa, Y. (2007). Modeling Decisions: Information Infusion and Aggregation Operations. Berlin Heidelberg: Springer-Verlag.

[25] Wang, T., \& Lee, H. (2009). Developing a fuzzy TOPSIS approach based on subjective weights and objective weights. Expert Systems with Applications, 36, 8980-8985.

[26] Wang, Y. M., \& Parkan, C. (2005). A minimax disparity approach for obtaining OWA operator weights. Information Sciences, 175, 20-29. 
[27] Xu, Z. (2005). An overview of methods for determining OWA weights. International Journal of Intelligent Systems, 20, 843-865.

[28] Yager, R. R. (1988). On ordered weighted averaging qggregation operators in multi-criteria decision making. IEEE Transactions on Systems, Man and Cybernetics, 18, 183-190.

[29] Yager, R. R. (1996). Quantifier guided aggregation using OWA operators. International Journal of Intelligent Systems, 11, 49-73.

[30] Yager, R. R., \& Kacprzyk, J. (1997). The Ordered Weighted Averaging Operators: Theory and Applications. Norwell, MA, US: Kluwer. 